\title{
Be careful .... She has a pituitary gland in her nose
}

\author{
N. M. Rabelink $\cdot$ P. Lips $\cdot$ J. A. Castelijns
}

Published online: 10 June 2011

(c) The Author(s) 2011. This article is published with open access at Springerlink.com

\begin{abstract}
In this case report we describe a 38 year-oldfemale with galactorrhea several months after the birth of an anencephalic child. She had hyperpolactemia and imaging of the pituitary gland revealed a midline defect and a nasopharyngeal mass compatible with a meningo(hypophyso-) encephalocele and possibly an ectopic teratoma or desmoid. She was treated with dopamine agonists for 10 years and after cessation of therapy her prolactin levels remain normal. The nasopharyngeal mass remained unchanged over time and there were no signs of hypopituitarism. The hyperprolactinemia at presentation was probably caused by earlier pregnancy and stalk dysfunction due to traction by the mass. With decline of pituitary size, after starting dopamine agonists, the traction probably reduced resulting in a normal prolactin level. Our patient was warned against manipulation in de nose, because this could damage the meningo-encephalocele. An MRI will be preformed every 2 years to monitor changes in de mass.
\end{abstract}

Keywords Hyperprolactinemia - Midline defect . Meningo-encephalocele $\cdot$ Pituitary gland · Pregnancy · Anencephalic

A 38 year-old-female consulted our policlinic because of hyperprolactinemia for which she had been treated with

\footnotetext{
N. M. Rabelink $(\bowtie) \cdot$ P. Lips

Department of Internal Medicine, Section Endocrinology,

VU Medical Centre, Postbox 7057, 1007 MB Amsterdam,

The Netherlands

e-mail:nrabelink@hotmail.com

\section{J. A. Castelijns}

Department of Radiology, VU Medical Centre, Amsterdam, The Netherlands
}

dopamine-agonists for nearly 10 years. Several months after giving birth to an anencephalic child she developed galactorrhea and hyperprolactinemia was diagnosed [Serum prolactin 1,645 mU/L (maximum reference value
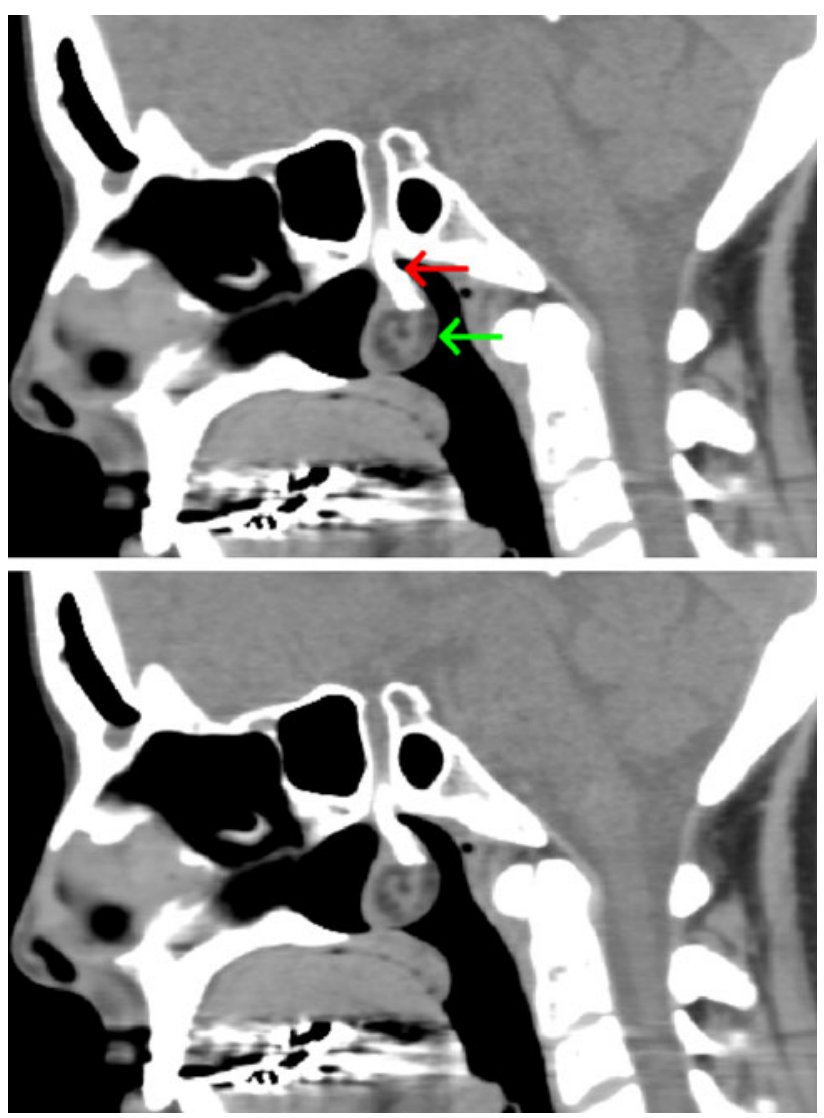

Fig. 1 Midsagittal computer tomography of the head. A heterogeneous nasopharyngeal mass (green arrow) is seen cranially connected by a channel. In this channel a calcified object can be seen (red arrow) 

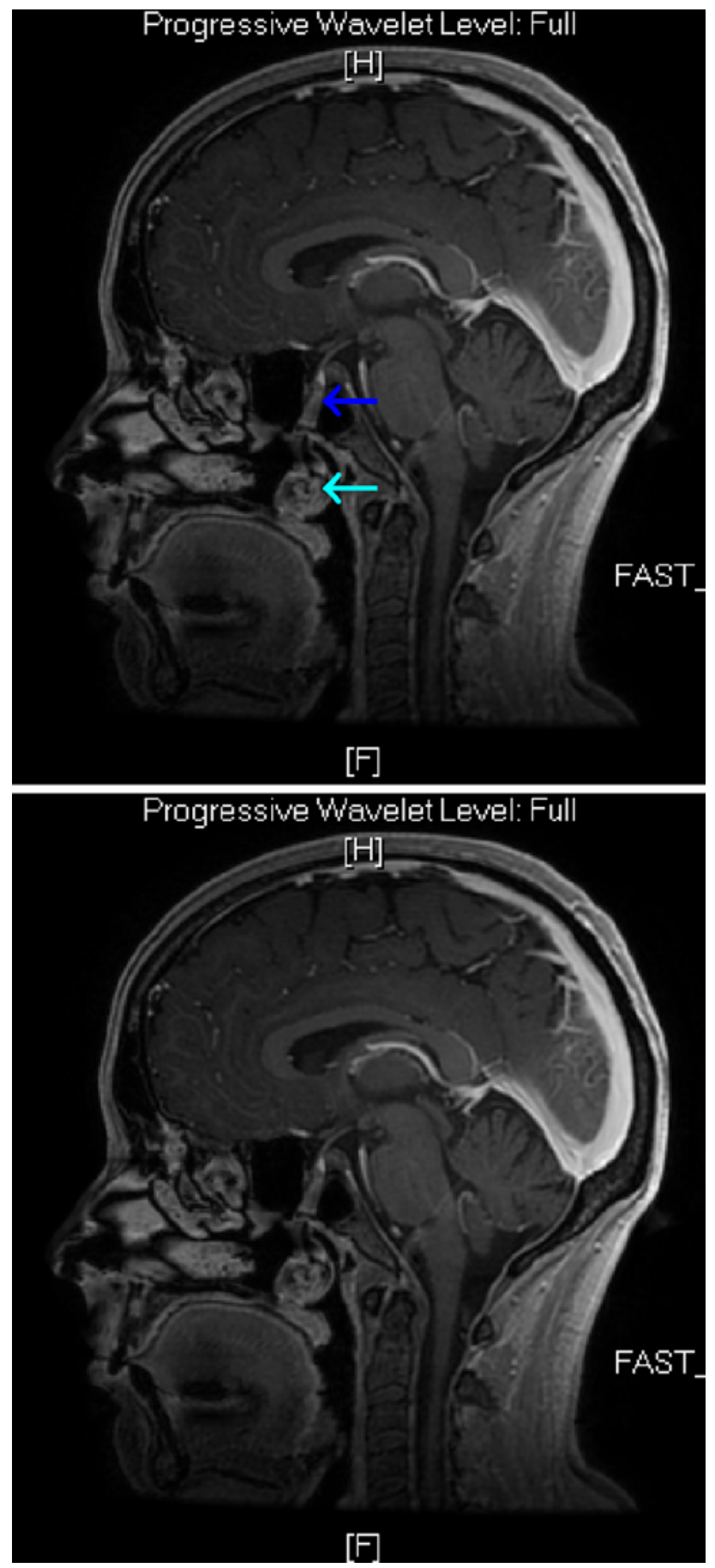

Fig. 2 Midsagittal T1SE MR image after contrast administration of the head. A lesion is seen extending from the hypophyseal region through a bony channel into the nasopharyngeal region (light blue arrow). Linear calcification is seen (dark blue arrow)

$450 \mathrm{mU} / \mathrm{L})]$. Imaging at that time showed a midline defect and nasopharyngeal mass. At presentation in our hospital her prolactin level was normal and there were no signs of hypopituitarism. The cabergoline she used was finished and her prolactin levels remain normal without medication.

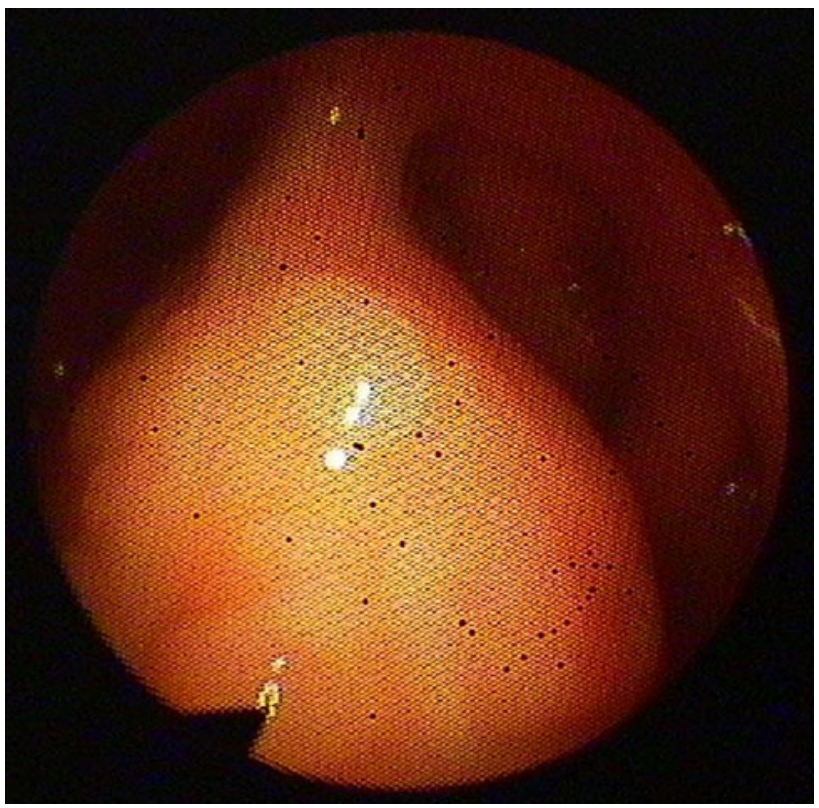

Fig. 3 Anterior rhinoscopy; Naso-pharyngeal mass

Midsagittal computer tomography of the head (Fig. 1), showed a heterogeneous mass $(20 \times 17 \mathrm{~mm})$ in the nasopharynx (green arrow). It appears to be cranially connected by a channel, bordered by cortical bone, with intracranial parts and is reaching caudally to the palatum molle at the choana. The sella turcica can not be recognized. Caudally in this channel a linear calcified object can be seen consistent with possibly a teratoma (red arrow). Midsagittal T1SE MR image after contrast administration of the head (Fig. 2) shows a lesion extending from the hypophyseal region through a bony channel, which is located in the central part of the sphenoid sinus, into the nasopharyngeal region (light blue arrow). The pituitary gland cannot be identified. Calcification is seen with linear low signal intensity (dark blue arrow). The remaining part of the lesion is enhancing heterogeneously. The images suggest a meningo-(hypophyso-) encephalocele, with possibly an ectopic teratoma or desmoid. The mass was visible when anterior rhinoscopy was performed (Fig. 3). Because biopsy or excision of the mass can cause hypopituitarism and the images of the mass remain unchanged over time, we decided to only perform an MRI-scan every 2 years to evaluate changes. Also patient was warned against manipulation in her nose to avoid damaging the meningo(hypophyso-) encephalocele.

The hyperprolactinemia at presentation was probably due to the prior pregnancy and possibly stalk dysfunction due to traction on the pituitary stalk of the mass. With decline of the pituitary size after starting dopamine agonists, the traction probably reduced resulting in a normal prolactin level. Midline defects causing a meningo-(hypophyso-) encephalocele 
in the naso-pharynx are very rare [1-3]. Transsphenoidal encephaloceles may only occur in 1 in 700.000 live births [2]. We are currently investigating a genetic cause for the midline defect in this patient.

\section{Conflict of interest None.}

Open Access This article is distributed under the terms of the Creative Commons Attribution Noncommercial License which permits any noncommercial use, distribution, and reproduction in any medium, provided the original author(s) and source are credited.

\section{References}

1. Hoving EW (2000) Nasal encephaloceles. Childs Nerv Syst 16:702-706

2. Knopp U, Knopp A, Stellmacher F, Reusche E, Loning M, Kantelhardt SR, Domarus H, Arnold H, Giese A (2009) A nonmidline spheno-orbital encephalocele in a newborn. Cen Eur Neurosurg 70:43-47

3. Yoshimoto Y, Noguchi M, Tsutsumi Y (1992) A case of transethmoidal encephalocele. No Shinkei Geka 20:249-254 